Original Research Paper

\title{
Effect of Repeated Freezing and Thawing on Nguni Sperm Parameters Evaluated by Computer Assisted Sperm Analyzer System
}

\author{
${ }^{1,3}$ Masindi Lottus Mphaphathi* and ${ }^{1,2}$ Tshimangadzo Lucky Nedambale \\ ${ }^{1}$ Agricultural Research Council, Animal Production, \\ Germplasm Conservation and Reproductive Biotechnologies, Private Bag X 2, Irene, 0062, RSA \\ ${ }^{2}$ Department of Animal Sciences, Faculty of Science, \\ Tshwane University of Technology, Private Bag X 680, Pretoria, 0001, RSA \\ ${ }^{3}$ Department of Animal, Wildlife and Grassland Sciences, \\ University of the Free State, P.O. Box 339, Bloemfontein, 9300, RSA
}

\begin{abstract}
Article history
Received: 26-08-2020

Revised: 16-01-2021

Accepted: 16-01-2021

Corresponding Author: Masindi Lottus Mphaphathi Agricultural Research Council, Animal Production,

Germplasm Conservation and Reproductive Biotechnologies, Private Bag X 2, Irene, 0062, RSA

Email: masindim@arc.agric.za
\end{abstract}

\begin{abstract}
The aims of the current study were to assess the effect of double freezing and thawing on Nguni semen parameters. Semen was collected from Nguni bulls. The Sperm Class Analyzer ${ }^{\circledR}$ system was used to evaluate sperm motility rates prior and post freezing, repeatedly. Semen samples were diluted with egg yolk citrate extender. Cooled semen samples were loaded into straws and placed inside the programmable freezer for freezing. The frozen semen straws were stored inside liquid nitrogen tank. The frozen semen straws were then thawed at $15^{\circ} \mathrm{C}\left(1^{\text {st }}\right.$ and $2^{\text {nd }}$ freezing and thawing followed the same process) for a duration of $5 \mathrm{~min}$ and further assessed post-thawed at 0 and 15 min during in vitro liquid storage at $15^{\circ} \mathrm{C}$. The treatment means were analyzed using Fisher's protected t-test least. There was no significant differences recorded on raw semen sperm total motility $(93.2 \%)$ and $1^{\text {st }}$ frozen-thawed at $0 \mathrm{~min}(82.6 \%)$, with recovery rate of $88.6 \%$ of the sperm total motility. There was a drastic decline observed in sperm total motility during the $1^{\text {st }}$ frozen-thaw at $15 \mathrm{~min}(77.6 \%), 2^{\text {nd }}$ frozen-thaw at $0 \mathrm{~min}$ $(31.3 \%)$ and $2^{\text {nd }}$ frozen-thaw at $15 \mathrm{~min}(30.1 \% ; P<0.05)$. In conclusion, the freezing and thawing process did not decrease the bull's sperm total motility percentage during the $1^{\text {st }}$ freezing and thawing process, as compared with raw semen. However, a drastic decline was observed during the $2^{\text {nd }}$ freezing-thawing process, as compared with raw semen.
\end{abstract}

Keywords: Bulls, Semen, Cryopreservation

\section{Introduction}

Cryopreservation of Nguni semen has not been extensively researched and cryo-banking of their genetic materials can provide an effective technique of maintaining their superior male genetic material for future use (Morrell and Mayer, 2017). The challenge of liquid nitrogen shortages and lack of liquid nitrogen supply to some parts of the country or areas on time may compromise the entire frozen semen straws and lead to the entire batch being discarded. Therefore, it is necessary to find new methods to reduce the amount of frozen straws that can be lost in batches, in cases where there is no adequate supply of liquid nitrogen in time. The double freezing and thawing process of semen could Abe done until the liquid nitrogen is delivered without a significant loss of sperm motility rates following the first thawing and in vitro liquid preservation. The semen banks or research institution can be able to restore the superior gamete in case when there is a delay or temporally no supply of liquid nitrogen gas in time.

The effectiveness of in vitro liquid perseveration for longer periods will also depend on the initial sperm cryoeffectiveness (Mphaphathi et al., 2016), type of semen extender, thawing temperature, storage temperature and semen re-dilution (Rodriguez et al., 1975). It has become vital to quantify bull semen parameters assessed by the computer assisted sperm analyzer system, as an attempt to get objective results pre and post freezing. It is essential for the cattle artificial insemination (AI) centers and cryo-biologist to obtain uniformly comparable results, where semen evaluators can make decisions. 
Different thawing temperatures have resulted in different motility results on sperm rates post-thaw. Avian egg yolk has been used as one of the important ingredients in extenders for bull semen freezing since 1939 (Amirat et al., 2005) and it remains important, globally. Obtaining the sexed sperm from previously frozen semen straws (reverse-sorted semen) provides an important role for the possibility of utilizing the semen of bulls with good genetic attributes that have died or become infertile but from whom frozen semen is still available (Morotti et al., 2014).

We hypothesized that Nguni bull semen can be successfully frozen and thawed repeatedly with only marginal changes in sperm motility quality as compared to the control sample. Therefore, the aims of the current study were to evaluate the effects of double freezing and thawing on wide range of bull sperm motility and velocity rates using the computeraided sperm analysis system.

\section{Materials and Methods}

\section{Chemicals and Reagents}

Chemicals and reagents were purchased from Sigma Chemical Co. (St. Louis, MO, USA), unless otherwise stated. All chemicals were of analytical grade.

\section{Experimental Nguni Bulls}

Experimental bulls $(\mathrm{n}=04)$ were cared for, according to the guidelines of the Agricultural Research Council (ARC)-Animal Production ethics committee (Ref: APIEC16/011). The Nguni bulls (aged 6 and 8 years) used in this trial were born from a pure bred Nguni breed cattle herd and were kept at the animal production-Loskop farm of the ARC in South Africa. Bulls were kept in camps and grazed on natural pasture as conservation breed with utilization. Veld condition of the grazing camps were monitored and rotation was done, based on the amount of forage available. All general cattle farm management and husbandry procedures were practiced.

\section{Individual Nguni Bulls Semen Collection and Characterization}

The sheath of each bull was cleaned prior to semen collection. Hygienic measures were practiced to avoid semen contamination. The electro ejaculator was used to collect semen from Nguni bulls $(\mathrm{n}=04)$ of known and proven fertility into a $15 \mathrm{~mL}$ Falcon tube. Following collection, the semen was transferred to a thermo-flask and maintained at $37^{\circ} \mathrm{C}$ for further evaluation.

Bull semen volume $(\mathrm{mL})$, sperm concentration $\left(\times 10^{6}\right)$ and the $\mathrm{pH}$ was evaluated as previously stated (Seshoka et al., 2016). The trial was repeated six times. The sperm total motility (TM) was further classified into rapid (RAP), medium (MED) or slow motility and progressive (PM) and non-progressive motility (NPM). The sperm Velocity rates measured included the curvilinear (VCL), straight line (VSL) and average path velocity (VAP), linearity (LIN), straightness (STR), wobble (WOB), amplitude of lateral head displacement (ALH), beat cross frequency (BCF) and hyperactivity (HPA). The settings for the Sperm Class Analyzer ${ }^{\circledR}\left(\mathrm{SCA}^{\circledR}\right)$ system used to assess the Nguni bull sperm motility and velocity parameters is shown in Table 1.

\section{Double Freezing and Thawing on Nguni Bull Semen}

During the $1^{\text {st }}$ freezing, individual semen samples were diluted with an Egg Yolk Citrate (EYC; fraction A) extender (Sodium citrate, Glucose, sterile water, egg yolk and Gentamycin). The diluted semen samples were equilibrated for $2 \mathrm{~h}$ at $5^{\circ} \mathrm{C}$ in the fridge. At the end of the equilibration, the individual semen sample was diluted further with EYC-fraction B (containing 12\% Glycerol cryoprotectant) extender. Cooled semen samples were further equilibrated for an additional $2 \mathrm{~h}$ at $5^{\circ} \mathrm{C}$. The semen samples were filled into $0.25 \mathrm{~mL}$ French straws, closed up with polyvalent powder and frozen using a programmable freezer (CBS, USA) connected to portable laptop freezer from 5 to $-5^{\circ} \mathrm{C}$ at $0.008^{\circ} \mathrm{C}$ min then from 4 to $130^{\circ} \mathrm{C}$ at $6^{\circ} \mathrm{C}$ min. Straws were immediately plunged directly into a liquid nitrogen $\left(-196^{\circ} \mathrm{C}\right)$ tank for storage until use ( $1^{\text {st }}$ freezing) (Seshoka et al., 2016).

Table 1: The $\mathrm{SCA}^{\circledR}$ (V.6.3.3.51) settings used to analyze the bull sperm motility and velocity parameters

\begin{tabular}{ll}
\hline Parameters & Settings \\
\hline Calibration name & $10 \mathrm{x}$ \\
Capture method & $\mathrm{Ph}-$ \\
Grid distance $(\mu \mathrm{m})$ & 100 \\
Box size (pixels) & 150 \\
Frame rate (fps) & 50 \\
Resolution & Normal \\
Style & Automatic \\
Disposable & Cover slide \\
Chamber depth $(\mu \mathrm{m})$ & 103 \\
Area $\left(\mu \mathrm{m}^{2}\right)(\min )$ & 5 \\
Area $\left(\mu \mathrm{m}^{2}\right)(\max )$ & 70 \\
Drifting $(\mu \mathrm{m} / \mathrm{s})$ & 10 \\
Static $(\mu \mathrm{m} / \mathrm{s})<$ & 10 \\
Slow-Medium $(\mu \mathrm{m} / \mathrm{s})$ & 25 \\
Rapid $(\mu \mathrm{m} / \mathrm{s})>$ & 100 \\
Progressive $(\mathrm{STR}>)$ & 70 \\
Connectivity $($ pixels $)$ & 12 \\
VAP points $($ pixels $)$ & 7 \\
\hline
\end{tabular}


For $1^{\text {st }}$ thawing: The frozen semen straws were removed from the liquid nitrogen tank $\left(-196^{\circ} \mathrm{C}\right)$ and exposed for $5 \mathrm{~min}$ at $15^{\circ} \mathrm{C}$ (our preliminary study showed that $15^{\circ} \mathrm{C}$ was the suitable thawing temperature for double freezing). The sperm motility and velocity rates were assessed at 0 and 15 min during liquid semen storage in vitro at $15^{\circ} \mathrm{C}$.

For $2^{\text {nd }}$ freezing (same as described in $1^{\text {st }}$ freezing), at the end of the $15 \mathrm{~min}$ (from previous $1^{\text {st }}$ thawing), cooled semen was then re-loaded into French straws and sealed with a polyvalent powder and frozen with a programmable freezer.

For $2^{\text {nd }}$ thawing (same as during $1^{\text {st }}$ thawing) the frozen semen straws were removed from the liquid nitrogen tank $\left(-196^{\circ} \mathrm{C}\right)$ and placed at $15^{\circ} \mathrm{C}$ for $5 \mathrm{~min}$. The sperm motility and velocity rates were evaluated (as previously described) at 0 and 15 min during liquid semen storage in vitro at $15^{\circ} \mathrm{C}$. The trial was repeated six times.

\section{Statistical Analysis}

Data were analyzed using the statistical programme GenStat at a significance level $(P<0.05)$. Analysis of variance was used to establish differences in the effect of semen, sperm motility and velocity parameters. Treatment means were separated using the
Fisher's protected t-test least significant difference. The data was presented as mean \pm standard deviation (SAS, 1999).

\section{Results}

\section{Individuals Bulls Semen Collection and Characterization}

The average Nguni bull ejaculated semen volume ranged from 3.3 to $4.4 \mathrm{~mL}$, while the semen $\mathrm{pH}$ ranged from 6.9 to 7.2 and the sperm cell concentration varied between 37.9 and $59.2 \times 10^{6}$ sperm/mL (Table 2). Numerically, Bull IDI 08-171 recorded a slightly higher semen volume $(4.4 \mathrm{~mL})$ than the other donors $(P>0.05)$. Furthermore, Bull IDI 06-11 recorded a slightly higher sperm concentration $\left(59.2 \times 10^{6} \mathrm{sperm} / \mathrm{mL}\right)$, when compared to the other donors $(P>0.05)$.

\section{Effect of Double Freezing and Thawing on Nguni Bull Sperm Motility Rates}

The individual's fresh semen sperm total motility ranged from 92.3 to $95.6 \%$, PM from 37.3 to $50.3 \%$ and RAP from 82.1 to $84.7 \%$ among the semen donors (Table 3).

Table 2: Individual characterization of ejaculated semen volume, semen $\mathrm{pH}$ and sperm concentration of Nguni bulls

\begin{tabular}{llll}
\hline Bull No & Semen volume $(\mathrm{mL})$ & Semen $\mathrm{pH}$ & $\begin{array}{l}\text { Sperm concentration } \\
\left(\times 10^{6} \mathrm{sperm} / \mathrm{mL}\right)\end{array}$ \\
\hline IDI 06-11 & $3.4 \pm 0.8$ & $7.1 \pm 0.2$ & $59.2 \pm 24.9$ \\
IDI 06-88 & $3.6 \pm 2.0$ & $7.2 \pm 0.3$ & $37.9 \pm 12.5$ \\
IDI 08-48 & $3.3 \pm 1.7$ & $6.9 \pm 0.2$ & $57.0 \pm 23.9$ \\
IDI 08-171 & $4.4 \pm 2.5$ & $7.0 \pm 0.3$ & $43.8 \pm 14.1$ \\
Average & $3.7 \pm 1.8$ & $7.1 \pm 0.3$ & $49.5 \pm 18.9$ \\
\hline
\end{tabular}

Difference not significant within a column $(P>0.05)$.

Table 3: Effect of $1^{\text {st }}$ and $2^{\text {nd }}$ freezing and thawing on diluted sperm parameters following repeated semen cryopreservation of Nguni bulls

\begin{tabular}{|c|c|c|c|c|c|c|c|c|c|}
\hline Bull No & Semen & $\mathrm{TM}(\%)$ & RR $(\%)$ & $\mathrm{PM}(\%)$ & NPM $(\%)$ & $\operatorname{RAP}(\%)$ & $\operatorname{MED}(\%)$ & SLW (\%) & STC $(\%)$ \\
\hline \multirow[t]{5}{*}{ IDI 06-11 } & Fresh semen (control) & $92.5 \pm 1.5^{\mathrm{a}}$ & - & $50.3 \pm 13.6^{\mathrm{a}}$ & $42.3 \pm 14.0^{\mathrm{b}, \mathrm{c}}$ & $82.1 \pm 7.3^{\mathrm{a}}$ & $9.7 \pm 6.0^{\mathrm{b}}$ & $0.9 \pm 0.8^{\mathrm{a}}$ & $7.5 \pm 1.6^{\mathrm{b}}$ \\
\hline & $1^{\mathrm{st}} \mathrm{F} / \mathrm{T}$ at $0 \mathrm{~min}$ & $88.0 \pm 14.2^{\mathrm{a}}$ & 95.2 & $25.4 \pm 14.8^{b, c}$ & $62.6 \pm 13.5^{\mathrm{a}}$ & $14.6 \pm 19.8^{b}$ & $29.2 \pm 13.9^{\mathrm{a}}$ & $44.2 \pm 23.2^{\mathrm{a}}$ & $13.6 \pm 17.3^{\mathrm{b}}$ \\
\hline & $1^{\text {st }} \mathrm{F} / \mathrm{T}$ at $15 \mathrm{~min}$ & $77.3 \pm 14.9^{\mathrm{a}}$ & 83.6 & $27.5 \pm 21.5^{\mathrm{a}}$ & $49.8 \pm 14.2^{\mathrm{a}, \mathrm{b}}$ & $13.3 \pm 16.7^{\mathrm{b}}$ & $26.8 \pm 16.7^{\mathrm{a}}$ & $37.3 \pm 21.5^{\mathrm{a}}$ & $22.7 \pm 14.9^{\mathrm{b}}$ \\
\hline & $2^{\text {nd }} \mathrm{F} / \mathrm{T}$ at $0 \mathrm{~min}$ & $37.8 \pm 18.0^{\mathrm{b}}$ & 40.9 & $5.4 \pm 5.1^{\mathrm{c}}$ & $32.4 \pm 13.0^{\mathrm{b}, \mathrm{c}}$ & $1.9 \pm 2.7^{\mathrm{b}}$ & $6.9 \pm 6.9^{\mathrm{b}}$ & $29.1 \pm 10.1^{\mathrm{a}}$ & $62.2 \pm 18.0^{\mathrm{a}}$ \\
\hline & $2^{\text {nd }} \mathrm{F} / \mathrm{T}$ at $15 \mathrm{~min}$ & $32.2 \pm 19.6^{\mathrm{b}}$ & 34.8 & $7.7 \pm 9.5^{\mathrm{b}, \mathrm{c}}$ & $24.5 \pm 10.1^{\mathrm{c}}$ & $2.3 \pm 4.5^{\mathrm{b}}$ & $7.7 \pm 8.7^{b}$ & $22.1 \pm 6.6^{\mathrm{a}, \mathrm{b}}$ & $67.9 \pm 19.6^{\mathrm{a}}$ \\
\hline \multirow[t]{4}{*}{ IDI 06-88 } & Fresh semen (control) & $92.3 \pm 2.3^{\mathrm{a}}$ & - & $37.3 \pm 12.9^{\mathrm{a}, \mathrm{b}}$ & $55.1 \pm 10.7^{\mathrm{a}}$ & $83.8 \pm 4.7^{\mathrm{a}}$ & $7.8 \pm 7.8^{\mathrm{a}}$ & $0.8 \pm 0.6^{\mathrm{d}}$ & $7.8 \pm 2.3^{\mathrm{c}}$ \\
\hline & $1^{\mathrm{st}} \mathrm{F} / \mathrm{T}$ at $0 \mathrm{~min}$ & $65.3 \pm 15.6^{\mathrm{b}}$ & 70.7 & $15.2 \pm 7.1^{\mathrm{b}}$ & $50.2 \pm 8.4^{\mathrm{a}}$ & $2.9 \pm 0.3^{\mathrm{b}}$ & $19.9 \pm 13.0^{\mathrm{a}}$ & $42.6 \pm 2.3^{b}$ & $34.7 \pm 15.6^{b}$ \\
\hline & $1^{\text {st }} \mathrm{F} / \mathrm{T}$ at $15 \mathrm{~min}$ & $76.9 \pm 5.0^{\mathrm{ab}}$ & 83.1 & $14.9 \pm 5.0^{\mathrm{b}}$ & $62.0 \pm 0.0^{\mathrm{a}}$ & $1.9 \pm 02.3^{\mathrm{b}}$ & $20.0 \pm 5.6^{\mathrm{a}}$ & $55.1 \pm 3.0^{\mathrm{a}}$ & $23.2 \pm 5.0^{\mathrm{b}, \mathrm{c}}$ \\
\hline & $2^{\text {nd }} \mathrm{F} / \mathrm{T}$ at $15 \mathrm{~min}$ & $16.9 \pm 1.8^{\mathrm{c}}$ & 18.3 & $1.6 \pm 0.4^{\mathrm{b}}$ & $15.4 \pm 1.5^{\mathrm{b}}$ & $0.7 \pm 0.1^{\mathrm{b}}$ & $1.3 \pm 1.1^{\mathrm{a}}$ & $14.9 \pm 0.8^{\mathrm{c}}$ & $83.1 \pm 1.8^{\mathrm{a}}$ \\
\hline \multirow[t]{5}{*}{ IDI $08-48$} & Fresh semen (control) & $92.4 \pm 1.4^{\mathrm{a}}$ & - & $39.7 \pm 13.8^{\mathrm{a}}$ & $52.7 \pm 15.1^{\mathrm{a}, \mathrm{b}}$ & $84.7 \pm 6.0^{\mathrm{a}}$ & $6.9 \pm 4.7^{b}$ & $0.8 \pm 0.6^{\mathrm{b}}$ & $7.6 \pm 1.4^{\mathrm{c}}$ \\
\hline & $1^{\text {st }} \mathrm{F} / \mathrm{T}$ at $0 \mathrm{~min}$ & $84.0 \pm 13.5^{\mathrm{a}}$ & 90.9 & $24.1 \pm 10.5^{\mathrm{a}, \mathrm{b}}$ & $60.0 \pm 4.5^{\mathrm{a}}$ & $20.1 \pm 28.8^{\mathrm{b}}$ & $25.2 \pm 7.0^{\mathrm{a}}$ & $38.8 \pm 21.6^{\mathrm{b}}$ & $16.0 \pm 13.5^{\mathrm{c}}$ \\
\hline & $1^{\mathrm{st}} \mathrm{F} / \mathrm{T}$ at $15 \mathrm{~min}$ & $68.5 \pm 10.5^{\mathrm{a}, \mathrm{b}}$ & 74.1 & $20.8 \pm 11.9^{a, b}$ & $47.7 \pm 5.3^{\mathrm{a}, \mathrm{b}}$ & $11.8 \pm 17.2^{\mathrm{b}}$ & $17.0 \pm 4.5^{\mathrm{a}, \mathrm{b}}$ & $40.0 \pm 11.1^{\mathrm{b}}$ & $31.5 \pm 10.5^{\mathrm{b}, \mathrm{c}}$ \\
\hline & $2^{\text {nd }} \mathrm{F} / \mathrm{T}$ at $0 \mathrm{~min}$ & $36.1 \pm 22.4^{c}$ & 39.1 & $5.7 \pm 4.8^{\mathrm{b}}$ & $30.5 \pm 18.2^{\mathrm{b}}$ & $2.6 \pm 3.6^{\mathrm{b}}$ & $7.0 \pm 5.7^{\mathrm{b}}$ & $26.5 \pm 14.8^{\mathrm{b}}$ & $63.8 \pm 22.3^{\mathrm{a}}$ \\
\hline & $2^{\text {nd }} \mathrm{F} / \mathrm{T}$ at $15 \mathrm{~min}$ & $40.6 \pm 24.8^{\mathrm{b}, \mathrm{c}}$ & 43.9 & $8.2 \pm 10.1^{\mathrm{b}}$ & $32.4 \pm 14.8^{\mathrm{b}}$ & $3.0 \pm 4.0^{\mathrm{b}}$ & $12.7 \pm 17.6^{\mathrm{a}, \mathrm{b}}$ & $25.0 \pm 5.0^{\mathrm{b}}$ & $59.4 \pm 24.8^{\mathrm{a}, \mathrm{b}}$ \\
\hline \multirow[t]{4}{*}{ IDI 08-171 } & Fresh semen (control) & $95.6 \pm 4.7^{\mathrm{a}}$ & - & $49.3 \pm 8.4^{\mathrm{a}}$ & $44.0 \pm 8.6^{\mathrm{a}}$ & $84.6 \pm 1.7^{\mathrm{a}}$ & $8.0 \pm 4.1^{\mathrm{c}}$ & $2.9 \pm 3.9^{\mathrm{c}}$ & $4.4 \pm 4.7^{\mathrm{b}}$ \\
\hline & $1^{\text {st }} \mathrm{F} / \mathrm{T}$ at $0 \mathrm{~min}$ & $85.6 \pm 7.6^{\mathrm{a}}$ & 89.5 & $23.8 \pm 9.0^{\mathrm{b}, \mathrm{c}}$ & $61.7 \pm 16.6^{\mathrm{a}}$ & $13.4 \pm 11.2^{\mathrm{b}}$ & $26.9 \pm 6.5^{\mathrm{a}, \mathrm{b}}$ & $45.3 \pm 22.5^{\mathrm{a}}$ & $14.4 \pm 7.5^{\mathrm{b}}$ \\
\hline & $2^{\text {nd }} \mathrm{F} / \mathrm{T}$ at $0 \mathrm{~min}$ & $29.1 \pm 16.8^{\mathrm{b}}$ & 30.4 & $7.4 \pm 6.0^{\mathrm{c}, \mathrm{d}}$ & $21.7 \pm 10.9^{b}$ & $2.2 \pm 2.8^{b}$ & $10.2 \pm 9.1^{\mathrm{b}, \mathrm{c}}$ & $16.8 \pm 6.6^{\mathrm{b}, \mathrm{c}}$ & $70.9 \pm 16.8^{\mathrm{a}}$ \\
\hline & $2^{\text {nd }} \mathrm{F} / \mathrm{T}$ at $15 \mathrm{~min}$ & $25.6 \pm 15.1^{\mathrm{b}}$ & 26.8 & $4.6 \pm 3.4^{d}$ & $21.0 \pm 12.6^{\mathrm{b}}$ & $2.7 \pm 3.7^{b}$ & $5.9 \pm 5.2^{\mathrm{c}}$ & $17.0 \pm 6.9^{\mathrm{b}, \mathrm{c}}$ & $74.4 \pm 15.1^{\mathrm{a}}$ \\
\hline
\end{tabular}

$\mathrm{TM}=$ total motility); $\mathrm{PM}=$ progressive motility; $\mathrm{NPM}=$ non-progressive motility; $\mathrm{RAP}=$ rapid $; \mathrm{MED}=$ medium; $\mathrm{SLW}=$ slow and $\mathrm{STC}=$ static 
Masindi Lottus Mphaphathi and Tshimangadzo Lucky Nedambale / American Journal of Animal and Veterinary Sciences 2021,16 (1): 1.6 DOI: 10.3844/ajavsp.2021.1.6

\begin{tabular}{|c|c|c|c|c|c|c|c|c|c|c|}
\hline Bull No & Semen & $\begin{array}{l}\text { VCL } \\
(\mu \mathrm{m} / \mathrm{sec})\end{array}$ & $\begin{array}{l}\text { VSL } \\
(\mu \mathrm{m} / \mathrm{sec})\end{array}$ & $\begin{array}{l}\text { VAP } \\
(\mu \mathrm{m} / \mathrm{sec})\end{array}$ & $\begin{array}{l}\text { LIN } \\
(\%)\end{array}$ & $\begin{array}{l}\text { STR } \\
(\%)\end{array}$ & $\begin{array}{l}\text { WOB } \\
(\%)\end{array}$ & $\begin{array}{l}\text { ALH } \\
(\mu \mathrm{m})\end{array}$ & $\begin{array}{l}\text { BCF } \\
(\mathrm{Hz})\end{array}$ & $\begin{array}{l}\text { HPA } \\
(\%)\end{array}$ \\
\hline \multirow[t]{5}{*}{ IDI 06-11 } & Fresh semen (control) & $152.9 \pm 21.8^{\mathrm{a}}$ & $74.0 \pm 17.6^{\mathrm{a}}$ & $116.0 \pm 17.8^{\mathrm{a}}$ & $48.4 \pm 9.6^{\mathrm{a}}$ & $63.5 \pm 9.0^{\mathrm{b}}$ & $75.9 \pm 4.6^{\mathrm{a}}$ & $2.7 \pm 0.8^{\mathrm{a}}$ & $20.3 \pm 2.2^{\mathrm{a}}$ & $10.9 \pm 5.0^{\mathrm{a}}$ \\
\hline & $1^{\text {st }} \mathrm{F} / \mathrm{T}$ at $0 \mathrm{~min}$ & $46.6 \pm 13.4^{b}$ & $22.4 \pm 10.2^{\mathrm{b}}$ & $30.3 \pm 11.1^{\mathrm{b}}$ & $47.1 \pm 8.5^{\mathrm{a}}$ & $72.9 \pm 6.4^{\mathrm{a}, \mathrm{b}}$ & $64.2 \pm 6.1^{\mathrm{b}}$ & $2.6 \pm 0.1^{\mathrm{a}}$ & $10.4 \pm 1.3^{\mathrm{b}}$ & $4.1 \pm 4.1^{b}$ \\
\hline & $1^{\mathrm{st}} \mathrm{F} / \mathrm{T}$ at $15 \mathrm{~min}$ & $46.0 \pm 16.3^{\mathrm{b}}$ & $23.1 \pm 10.7^{\mathrm{b}}$ & $30.3 \pm 12.4^{b}$ & $48.9 \pm 7.8^{\mathrm{a}}$ & $74.9 \pm 6.9^{\mathrm{a}}$ & $65.1 \pm 4.8^{\mathrm{b}}$ & $2.6 \pm 0.4^{\mathrm{a}}$ & $10.8 \pm 1.3^{b}$ & $3.1 \pm 3.2^{\mathrm{b}}$ \\
\hline & $2^{\text {nd }} \mathrm{F} / \mathrm{T}$ at $0 \mathrm{~min}$ & $27.0 \pm 5.5^{\mathrm{b}}$ & $12.5 \pm 2.6^{\mathrm{b}}$ & $17.3 \pm 2.9^{\mathrm{b}}$ & $46.4 \pm 4.1^{\mathrm{a}}$ & $72.1 \pm 5.0^{\mathrm{a}, \mathrm{b}}$ & $64.3 \pm 3.7^{\mathrm{b}}$ & $1.8 \pm 1.2^{\mathrm{a}}$ & $7.9 \pm 5.8^{\mathrm{b}}$ & $0.5 \pm 0.5^{\mathrm{b}}$ \\
\hline & $2^{\text {nd }} \mathrm{F} / \mathrm{T}$ at $15 \mathrm{~min}$ & $28.6 \pm 2.1^{\mathrm{b}}$ & $14.4 \pm 1.8^{\mathrm{b}}$ & $18.7 \pm 1.5^{\mathrm{b}}$ & $50.9 \pm 9.0^{\mathrm{a}}$ & $77.1 \pm 4.9^{\mathrm{a}}$ & $65.7 \pm 8.2^{\mathrm{b}}$ & $2.4 \pm 0.4^{\mathrm{a}}$ & $11.4 \pm 1.5^{b}$ & $0.9 \pm 1.6^{\mathrm{b}}$ \\
\hline \multirow[t]{4}{*}{ IDI 06-88 } & Fresh semen (control) & $185.5 \pm 27.6^{\mathrm{a}}$ & $85.9 \pm 35.2^{\mathrm{a}}$ & $117.5 \pm 31.3^{\mathrm{a}}$ & $45.5 \pm 12.2^{\mathrm{a}}$ & $71.7 \pm 10.9^{\mathrm{a}}$ & $62.8 \pm 7.5^{\mathrm{a}, \mathrm{b}}$ & $3.7 \pm 0.4^{\mathrm{a}, \mathrm{b}}$ & $25.0 \pm 2.8^{\mathrm{a}}$ & $12.8 \pm 6.6^{\mathrm{a}}$ \\
\hline & $1^{\mathrm{st}} \mathrm{F} / \mathrm{T}$ at $15 \mathrm{~min}$ & $40.1 \pm 9.5^{b}$ & $20.5 \pm 5.9^{b}$ & $26.8 \pm 6.9^{b}$ & $50.8 \pm 2.8^{\mathrm{a}}$ & $76.0 \pm 2.5^{\mathrm{a}}$ & $66.8 \pm 1.5^{\mathrm{a}}$ & $2.8 \pm 0.1^{\mathrm{a}, \mathrm{b}}$ & $11.8 \pm 1.3^{\mathrm{b}}$ & $1.3 \pm 1.6^{\mathrm{b}}$ \\
\hline & $2^{\text {nd }} \mathrm{F} / \mathrm{T}$ at $0 \mathrm{~min}$ & $25.9 \pm 2.8^{b}$ & $10.0 \pm 3.0^{\mathrm{b}}$ & $15.6 \pm 0.9^{b}$ & $38.1 \pm 7.8^{\mathrm{a}}$ & $63.5 \pm 16.1^{\mathrm{a}}$ & $60.4 \pm 2.9^{\mathrm{a}, \mathrm{b}}$ & $2.7 \pm 0.3^{\mathrm{b}}$ & $12.3 \pm 0.4^{b}$ & $0.0 \pm 0.0^{\mathrm{b}}$ \\
\hline & $2^{\text {nd }} \mathrm{F} / \mathrm{T}$ at $15 \mathrm{~min}$ & $26.5 \pm 3.7^{b}$ & $10.0 \pm 0.8^{\mathrm{b}}$ & $14.1 \pm 1.2^{\mathrm{b}}$ & $38.0 \pm 2.3^{\mathrm{a}}$ & $71.2 \pm 0.2^{\mathrm{a}}$ & $53.4 \pm 3.0^{\mathrm{b}}$ & $3.8 \pm 0.0^{\mathrm{b}}$ & $13.1 \pm 0.1^{\mathrm{b}}$ & $0.4 \pm 0.6^{\mathrm{b}}$ \\
\hline \multirow[t]{5}{*}{ IDI 08-48 } & Fresh semen (control) & $162.0 \pm 31.6^{\mathrm{a}}$ & $57.6 \pm 1.8^{\mathrm{a}}$ & $95.1 \pm 4.0^{\mathrm{a}}$ & $36.4 \pm 4^{\mathrm{b}}$ & $60.6 \pm 2.2^{\mathrm{b}}$ & $59.9 \pm 9.5^{\mathrm{a}}$ & $4.1 \pm 0.9^{\mathrm{a}}$ & $18.6 \pm 1.8^{\mathrm{a}}$ & $17.6 \pm 15.2^{\mathrm{a}}$ \\
\hline & $1^{\mathrm{st}} \mathrm{F} / \mathrm{T}$ at $0 \mathrm{~min}$ & $46.2 \pm 10.0^{\mathrm{b}}$ & $20.2 \pm 5.1^{\mathrm{b}}$ & $28.4 \pm 5.8^{\mathrm{b}}$ & $44.3 \pm 9.3^{\mathrm{a}, \mathrm{b}}$ & $71.3 \pm 8.6^{\mathrm{a}}$ & $61.6 \pm 5.6^{\mathrm{a}}$ & $2.8 \pm 0.3^{\mathrm{b}}$ & $10.3 \pm 1.9^{b}$ & $4.4 \pm 4.4^{\mathrm{a}, \mathrm{b}}$ \\
\hline & $1^{\mathrm{st}} \mathrm{F} / \mathrm{T}$ at $15 \mathrm{~min}$ & $40.4 \pm 5.0^{\mathrm{b}}$ & $19.6 \pm 2.4^{\mathrm{b}, \mathrm{c}}$ & $25.2 \pm 2.9^{\mathrm{b}, \mathrm{c}}$ & $48.7 \pm 1.8^{\mathrm{a}}$ & $78.0 \pm 3.5^{\mathrm{a}}$ & $62.5 \pm 0.8^{\mathrm{a}}$ & $2.8 \pm 0.2^{\mathrm{b}}$ & $11.7 \pm 1.3^{b}$ & $5.1 \pm 6.0^{\mathrm{a}, \mathrm{b}}$ \\
\hline & $2^{\text {nd }} \mathrm{F} / \mathrm{T}$ at $0 \mathrm{~min}$ & $30.0 \pm 3.3^{\mathrm{b}}$ & $13.9 \pm 1.5^{\mathrm{c}, \mathrm{d}}$ & $18.7 \pm 1.7^{\mathrm{c}, \mathrm{d}}$ & $46.3 \pm 1.2^{\mathrm{a}, \mathrm{b}}$ & $74.0 \pm 2.5^{\mathrm{a}}$ & $62.6 \pm 1.4^{\mathrm{a}}$ & $2.7 \pm 0.5^{b}$ & $9.2 \pm 3.0^{\mathrm{b}}$ & $0.5 \pm 0.3^{\mathrm{b}}$ \\
\hline & $2^{\text {nd }} \mathrm{F} / \mathrm{T}$ at $15 \mathrm{~min}$ & $28.9 \pm 4.6^{\mathrm{b}}$ & $12.8 \pm 3.8^{\mathrm{d}}$ & $17.8 \pm 4.3^{\mathrm{d}}$ & $43.7 \pm 6.4^{\mathrm{a}, \mathrm{b}}$ & $71.2 \pm 7.0^{\mathrm{a}}$ & $61.3 \pm 6.5^{\mathrm{a}}$ & $3.1 \pm 1.0^{\mathrm{a}, \mathrm{b}}$ & $9.8 \pm 5.0^{\mathrm{b}}$ & $0.8 \pm 0.8^{b}$ \\
\hline & $1^{\mathrm{st}} \mathrm{F} / \mathrm{T}$ at $0 \mathrm{~min}$ & $45.4 \pm 13.1^{\mathrm{b}}$ & $20.9 \pm 11.5^{b}$ & $28.5 \pm 11.2^{\mathrm{b}}$ & $43.9 \pm 10.9^{\mathrm{a}}$ & $70.6 \pm 10.3^{\mathrm{a}, \mathrm{b}}$ & $61.6 \pm 6.0^{c}$ & $2.8 \pm 0.1^{\mathrm{a}}$ & $10.7 \pm 2.7^{b}$ & $4.8 \pm 4.0^{\mathrm{a}, \mathrm{b}}$ \\
\hline & $1^{\mathrm{st}} \mathrm{F} / \mathrm{T}$ at $15 \mathrm{~min}$ & $46.9 \pm 15.2^{\mathrm{b}}$ & $27.7 \pm 11.8^{\mathrm{b}}$ & $33.3 \pm 12.5^{\mathrm{b}}$ & $58.1 \pm 7.6^{\mathrm{a}}$ & $82.3 \pm 5.3^{\mathrm{a}}$ & $70.2 \pm 4.8^{\mathrm{a}, \mathrm{b}}$ & $2.5 \pm 0.5^{\mathrm{a}}$ & $8.9 \pm 6.0^{\mathrm{b}}$ & $5.5 \pm 5.8^{\mathrm{a}, \mathrm{b}}$ \\
\hline & $2^{\text {nd }} \mathrm{F} / \mathrm{T}$ at $0 \mathrm{~min}$ & $33.4 \pm 12.1^{\mathrm{b}}$ & $14.7 \pm 5.4^{\mathrm{b}}$ & $20.2 \pm 7.4^{b}$ & $44.3 \pm 4.4^{\mathrm{a}}$ & $73.3 \pm 3.2^{\mathrm{a}, \mathrm{b}}$ & $60.4 \pm 3.5^{\mathrm{c}}$ & $2.3 \pm 0.3^{\mathrm{a}}$ & $10.5 \pm 1.8^{b}$ & $0.6 \pm 0.6^{\mathrm{b}}$ \\
\hline & $2^{\text {nd }} \mathrm{F} / \mathrm{T}$ at $15 \mathrm{~min}$ & $31.0 \pm 9.6^{\mathrm{b}}$ & $14.8 \pm 7.5^{b}$ & $19.8 \pm 7.2^{b}$ & $46.1 \pm 9.5^{\mathrm{a}}$ & $72.4 \pm 10.1^{\mathrm{a}, \mathrm{b}}$ & $63.2 \pm 4.0^{\mathrm{b}, \mathrm{c}}$ & $2.4 \pm 0.3^{\mathrm{a}}$ & $11.2 \pm 4.2^{b}$ & $0.7 \pm 0.8^{b}$ \\
\hline
\end{tabular}

$\overline{\mathrm{a}-\mathrm{d}}$ Values with different superscript within a column are significantly different $(P<0.05)$. Frozen/Thawed $=(\mathrm{F} / \mathrm{T})$. Difference between fresh $(\mathrm{control})$ and frozen semen within a bull.

$\mathrm{VCL}=$ curvilinear velocity; VSL = straight line velocity; VAP = average path velocity; LIN = linearity; STR = straightness; $\mathrm{WOB}=$ wobble and $\mathrm{ALH}=$ amplitude of lateral head displacement; $\mathrm{BCF}=$ beat cross frequency and HPA = hyperactive.

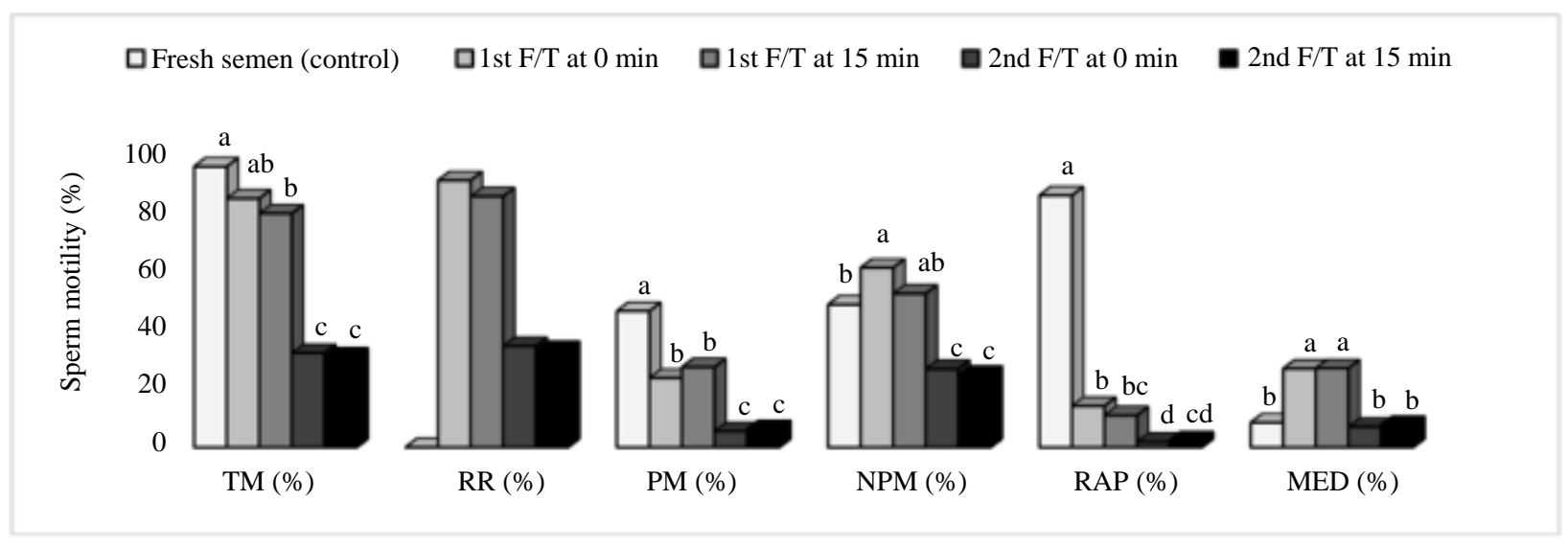

Fig. 1: The average sperm cryosurvival following double freezing and thawing of semen from Nguni bulls. ${ }^{\mathrm{a}-\mathrm{d}}$ Different superscript across the bars differ significantly $(P<0.05)$. Total motility $(\mathrm{TM})$, recovery rate $(\mathrm{RR})$, progressive motility (PM), nonprogressive motility (NPM), rapid (RAP) and medium (MED). Recovery rate was calculated as follows: Value after thawing/value before freezing $\times 100$

Following $1^{\text {st }}$ freezing and $1^{\text {st }}$ thawing of semen straws (Table 3), the sperm TM\% (ranged from 65.3 to 88.0); PM\% (15.2 to 25.4) and the RAP\% (2.9 to 20.1) decreased drastically between bulls and the groups (fresh semen and frozen-thawed semen) $(P<0.05)$ at $0 \mathrm{~min}$. There was individual difference recorded on sperm TM\% and PM\% as from 0 to 15 min post thawed $(P<0.05)$.

Following $2^{\text {nd }}$ freezing and $2^{\text {nd }}$ thawing of semen straws (Table 3), the sperm TM\% (ranged from 14.4 to 37.8); PM\% (1.4 to 7.4) and RAP\% (0.0 to 2.6) among the bulls $(P<0.05)$ at $0 \mathrm{~min}$. There was an individual difference observed on sperm TM\% and PM\% as from 0 to 15 min post thawed $(P<0.05)$. There was marked decline in the initial total sperm motility between fresh control and $2^{\text {nd }}$ frozen-thawed semen $(P<0.05)$.

\section{Effect of Double Freezing and Thawing on Nguni Bull Sperm Velocity Rates}

Following $1^{\text {st }}$ freezing and thawing of semen straws (Table 4), inconsistent on sperm LIN $(\%)$, VCL $(\mu \mathrm{m} / \mathrm{sec})$ and VSL $(\mu \mathrm{m} / \mathrm{sec})$ within the bulls was recorded $(P<0.05)$. During the $2^{\text {nd }}$ freezing and thawing of semen straws (Table 3), from 0 to $15 \mathrm{~min}$ had no effect $(P>0.05)$ on sperm ALH $(\mu \mathrm{m})$ and BCF $(\mathrm{Hz})$ within the individual. Velocity characteristics such as VCL and VAP $(\mu \mathrm{m} / \mathrm{sec})$ were reduced $(P<0.05)$, following $2^{\text {nd }}$ freezing and $2^{\text {nd }}$ thawing in all bulls semen. 
The Average Sperm Cryosurvival Following Double Freezing and Thawing of Nguni Bull Semen

No significant differences were recorded between the initial (fresh semen) total sperm motility\% (93.2\%) and the $1^{\text {st }} \mathrm{F} / \mathrm{T}$ at $0 \mathrm{~min}(82.6 \%)$ with the sperm $\mathrm{TM}$ rate recovery of $88.6 \%$ (Fig. 1). Furthermore, a drastic decline was observed in sperm TM during the $1^{\text {st }} \mathrm{F} / \mathrm{T}$ at $15 \mathrm{~min}(77.6 \%), 2^{\text {nd }} \mathrm{F} / \mathrm{T}$ at $0 \mathrm{~min}(31.3 \%)$ and $2^{\text {nd }} \mathrm{F} / \mathrm{T}$ at $15 \min (30.1 \%)(P<0.05)$. The average rapid sperm motility\% was reduced following the $1^{\text {st }} \mathrm{F} / \mathrm{T}(P<0.05)$, but remained persistent and steady between the treatment groups $(P>0.05)$.

\section{Discussion}

The study demonstrated that repeated freezing and thawing was possible for cryopreservation of semen from Nguni bulls. The freezing-thawing process did not reduce the Nguni bull sperm TM\% during the $1^{\text {st }}$ freezing and thawing process, as compared with raw semen. However, a significant drastic decline was recorded during the $2^{\text {nd }}$ freezing-thawing processes, as compared with both raw and $1^{\text {st }}$ freezing semen. Nguni bull sperm is one of the important genetic material for breed conservation programs. The present study was an attempt to demonstrate the current status on cryoeffectiveness of semen from Nguni bulls. To our knowledge, this was the first attempt to cryopreserve semen from Nguni bulls using this protocol (double/repeated freezing and thawing). For the past few decades, the bull semen cryopreservation technique has been reported to result in the loss of more than $40 \%$ of viable sperm during the freezing and thawing process and yet progress in cryopreservation techniques has been slow (Yoshida, 2000; Nagata et al., 2019). There was no significant differences observed between the initial (fresh semen) sperm $\mathrm{TM}$ and $1^{\text {st }} \mathrm{F} / \mathrm{T}$ at 0 min, with a sperm TM rate recovery of $88.6 \%$. Seshoka et al. (2016) found that semen post-thawing had drastically reduced the sperm velocity rates of bulls.

In addition, there has been a drastic decline recorded in sperm TM\% during the $1^{\text {st }} \mathrm{F} / \mathrm{T}$ at $15 \mathrm{~min}, 2^{\text {nd }} \mathrm{F} / \mathrm{T}$ at $0 \mathrm{~min}$ and $2^{\text {nd }} \mathrm{F} / \mathrm{T}$ at $15 \mathrm{~min}$. The $2^{\text {nd }} \mathrm{F} / \mathrm{T}$ at $15 \mathrm{~min}$ was simply impractical and sperm cannot be sustained in liquid preservation in vitro for more than $15 \mathrm{~min}$. The post-thaw sperm motility has always been a commonly used parameter for evaluation of frozen-thawed semen in bulls (Mathur et al., 2014; Khalil et al., 2018).

The post-thaw semen is expected to be deposited into the body of uterus of the cow within 15 min for better conception during AI. Individual variation was evidence in the Nguni semen donors used in the present study, with one bull able to have sperm TM of above $40 \%$ for both $1^{\text {st }} \mathrm{F} / \mathrm{T}$ at $15 \mathrm{~min}$ and $2^{\text {nd }} \mathrm{F} / \mathrm{T}$ at $15 \mathrm{~min}$. Therefore, time-dependent changes in sperm motility rates of bulls during in vitro liquid semen storage post thaw was also evident. The major source of variation in sperm motility rates between bulls is unknown.

The sperm VCL and VAP were reduced following $1^{\text {st }}$ $\mathrm{F} / \mathrm{T}$ and remained constant and stable between the treatment groups. Cryo-banking of semen from bulls is important for the beef cattle genetic industry and therefore, the cryopreservation protocol needs to be improved. In addition to its role, cryo-banking of semen from superior bulls is an essential backup for adequate insemination dosages in cases of diseases, infertility or death (Arav et al., 2002). Several structural and ultrastructural sperm components have been recorded as being compromized by the cryopreservation process (Khalil et al., 2018). Developing a protocol such as this, may prove useful for valuable bull semen when there is a shortage supply of liquid nitrogen.

\section{Conclusion}

This study demonstrated that the freezing-thawing process did not reduce sperm TM percentage in all the Nguni bulls during the $1^{\text {st }}$ freezing and thawing process, as compared with raw semen. However, a drastic decline on bull sperm TM percentage was recorded during the $2^{\text {nd }}$ freezing-thawing processes, as compared with raw semen.

\section{Acknowledgement}

The author wishes to acknowledge the Agricultural Research Council-Parliamentary Grant, the Department of Agriculture, Land Reform and Rural Development and the National Research Foundation for funding. The Germplasm Conservation and Reproductive Biotechnologies group is thanked for their support.

\section{Funding Information}

The study was supported by grants from the Agricultural Research Council-Parliamentary Grant, the Department of Agriculture Forestry and Fishery and the National Research Foundation.

\section{Author's Contributions}

All authors equally contributed in this work.

\section{Ethics}

Experimental bulls were cared for, according to the guidelines of the Agricultural Research Council (ARC)Animal Production ethics committee (Ref: APIEC16/011).

\section{Conflict of Interest}

All authors have reviewed and approved this final version of the manuscript. There is no competing interest in our submission. 


\section{References}

Amirat, L., Anton, M., Tainturier, D., Chatagnon, G., Battut, I., \& Courtens, J. L. (2005). Modifications of bull spermatozoa induced by three extenders: Biociphos, low density lipoprotein and Triladyl, before, during and after freezing and thawing. Reproduction, 129(4), 535-543.

Arav, A., Zeron, Y., Shturman, H., \& Gacitua, H. (2002). Successful pregnancies in cows following double freezing of a large volume of semen. Reproduction Nutrition Development, 42(6), 583-586.

Khalil, W. A., El-Harairy, M. A., Zeidan, A. E., Hassan, M. A., \& Mohey-Elsaeed, O. (2018). Evaluation of bull spermatozoa during and after cryopreservation: Structural and ultrastructural insights. International journal of veterinary science and medicine, 6, S49-S56.

Mathur, A. K., Kumar, S., Prabhakar, J. H., Mandal, D. K., Ingale, H. R., \& James, J. O. S. E. (2014). Motility of frozen thawed semen after thermal resistance test. Indian Journal of Animal Reproduction, 35(1), 18-20.

Morotti, F., Sanches, B. V., Pontes, J. H. F., Basso, A. C., Siqueira, E. R., Lisboa, L. A., \& Seneda, M. M. (2014). Pregnancy rate and birth rate of calves from a large-scale IVF program using reverse-sorted semen in Bos indicus, Bos indicus-taurus and Bos taurus cattle. Theriogenology, 81(5), 696-701.
Morrell, J. M., \& Mayer, I. (2017). Reproduction biotechnologies in germplasm banking of livestock species: a review. Zygote, 25(5), 545-557.

Mphaphathi, M. L., Seshoka, M. M., Luseba, D., Sutherland, B., \& Nedambale, T. L. (2016). The characterisation and cryopreservation of Venda chicken semen. Asian Pacific Journal of Reproduction, 5(2), 132-139.

Nagata, M. B., Egashira, J., Katafuchi, N., Endo, K., Ogata, K., Yamanaka, K., \& Yamashita, K. (2019). Bovine sperm selection procedure prior to cryopreservation for improvement of post-thawed semen quality and fertility. Journal of Animal Science and Biotechnology, 10(1), 91.

Rodriguez, O. L., Berndtson, W. E., Ennen, B. D., \& Pickett, B. W. (1975). Effect of rates of freezing, thawing and level of glycerol on the survival of bovine spermatozoa in straws. Journal of animal science, 41(1), 129-136.

SAS., 1999. SAS/STAT User's Guide. Version 8.

Seshoka, M. M., Mphaphathi, M. L., \& Nedambale, T. L. (2016). Comparison of four different permitting and combination of two best cryoprotectants on freezing Nguni sperm evaluated with the aid of computer aided sperm analysis. Cryobiology, 72(3), 232-238.

Yoshida, M. (2000). Conservation of sperms: Current status and new trends. Animal Reproduction Science, 60, 349-355. 